\title{
Device-Orientation is More Engaging than Drag (at Least in Mobile Computing)
}

\section{Mattias Arvola}

SICS East Swedish ICT AB

Department of Computer and

Information Science

Linköping University

SE-58381 Linköping, Sweden

mattias.arvola@liu.se

\section{Anna Holm}

Department of Computer and

Information Science

Linköping University

SE-58381 Linköping, Sweden

friidrottan@hotmail.com

Permission to make digital or hard copies of part or all of this work for personal or classroom use is granted without fee provided that copies are not made or distributed for profit or commercial advantage and that copies bear this notice and the full citation on the first page. Copyrights for thirdparty components the Owner/Author.

Copyright is held by the owner/author(s).

NordiCHI '14, Oct 26-30 2014, Helsinki, Finland

ACM $978-1-4503-2542-4 / 14 / 10$

http://dx.doi.org/10.1145/2639189.2670245

\begin{abstract}
Does device-orientation-based panning on mobile devices facilitate engagement? 20 users were asked to pan panoramas by turning around and changing the direction of the device, and by swiping with the finger on the touchscreen. The participants were also asked to rate how engaging they found it on the User

Engagement Scale. It turned out that deviceorientation-based panning was more engaging than drag-based panning. Moving your body to navigate information can pull you into an affective loop.
\end{abstract}

\section{Author Keywords}

Movement-based interaction; engagement; immersion; affective computing; navigation; panorama; deviceorientation-based panning; user experience.

\section{ACM Classification Keywords}

H5.1 Information interfaces and presentation:

Multimedia Information Systems: evaluation; H.5.2 User Interfaces.

\section{Introduction}

Device-orientation-based panning is an interaction technique where the user navigates a panorama or 3Dmodel by holding a device (mobile phone or tablet) in 


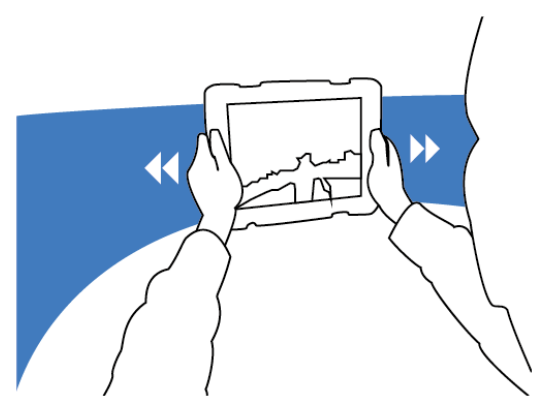

Figure 1. Device-direction-based panning of a panorama. The user holds the device in front of him or her and turns left or right to move the peephole over the panoramic image. front of him or her and turns the entire body. As the device and user change direction, the view of the mode or panorama also changes (Figure 1 ). It has been used instead of ordinary drag-based panning (i.e. swipe) with the design goal of engagement [e.g. 7]. The purpose of this study is to investigate the correctness of such a design decision.

We use the User Engagement Scale (UES) and define engagement as a user experience quality consisting of the following sub-dimensions: Focused Attention, Perceived Usability, Aesthetics, Endurability, Novelty, and Felt Involvement [8]. The UES is a questionnaire that measures engagement, but it can also be used to measure the sub-scales.

The research question addressed here is whether device-orientation-based panning actually facilitates more engagement than drag-based panning?

\section{Device-Orientation-based Panning}

Device-orientation based panning is an example of dynamic peephole navigation where the user move the peephole across the spatial layout (in this case the panoramic photo), while drag-based panning is an example of static peephole navigation where the user move the layout behind the static peephole. Dynamic peephole navigation has shown benefits over static peephole navigation in line length discrimination tasks [5].

Hürst and Bilyalov [2] compared the device-orientationbased panning with drag-based panning and found that device-orientation was twice as good in an orientation task, 75\% better in an object size discrimination task and was preferred by $80 \%$ of the users. Hürst and
Bilyalov also observed that users resorted to dragbased navigation if they were sitting.

A panorama can be coupled to the place the users currently are at. It can accordingly be on-site or offsite, which has shown effects on target acquisition in map navigation [3]. On-site coupling between a panorama and the real world is a form of augmented reality. In camera-based augmented reality, a virtual image is projected onto the camera view with aligned perspectives, or with views that zoom in, provides bird's view, map view, sub-view, fly-in view, free view, or snapshots [4]. A pre-captured panorama with device-orientation based panning and oriented according to the compass can, in some cases, also be used instead of the live camera view. Large differences between where the panorama was captured and where it is viewed reduce immersion and change the experience [9]. Techniques for clearly conveying the spatial relationship between the panorama and the environment may be necessary for on-site panoramas [6].

Device-orientation based panning means moving more of one's entire body, compared to using a touchscreen. We know from earlier research that more intensive movement-based interaction can give more engaging experiences [1]. We do, however, not know if this applies to the relatively low-intensive deviceorientation-based panning. The research question is if device-orientation-based panning on mobile devices facilitates engagement. Our hypothesis is that it does.

\section{Method}

A counterbalanced within subjects experiment with 20 participants was conducted. 


\section{Apparatus and Materials}

360Cities, a commercial application available on Apple App Store. It supports both device-orientation based panning and drag-based panning of cubical panoramas.

Apple iPad Mini with iOS 7.

UES for measuring engagement was measured using the UES. Three nonapplicable statements in the UES were removed, and small changes of wording were made (e.g. reading changed to viewing).

\section{Participants}

20 students, $14(70 \%)$ men and $6(30 \%)$ women participated. They were 21 to 26 years old ( $M=23.5$, $\mathrm{SD}=1.7)$. All were regular users of smartphones or tablets. 35\% used iOS, 65\% used Android, and 15\% used Windows. $65 \%$ had never created or watched panoramas. They were familiar with using a

touchscreen, but less familiar with using device orientation.

\section{Procedure}

Tests were held in a meeting room at our offices to minimize distractions. In the drag-based condition, the iPad stood in landscape mode on a stand in front of a standing participant, creating a situation similar to an information kiosk. The participants were instructed to use touch to explore panoramas of their own choice from the Featured category for five minutes. They could change panorama whenever they wanted. A UES questionnaire was then filled out. In the deviceorientation-based condition the participants were instructed to stand up and hold the iPad in their hands in landscape mode. They were instructed to move the device and turn around to explore panoramas. They could explore panoramas of their own choice from the Featured category for five minutes in each condition. They could change panorama whenever they wanted. A UES questionnaire was filled out after each test. A short post-test questionnaire was also administrated to gather reflections on the test from the participants. Paired-samples T-tests were calculated for mean UESscore and for the mean on the different sub-

dimensions. One-tailed tests were used since the hypothesis was directional. Cohen's d was calculated to measure effect size. Cronbach's alpha was calculated to measure reliability.

\section{Results}

One user was excluded from the experiment due to a feeling of motion sickness from the device-orientationbased condition. The results are accordingly based on nineteen participants (see Table 1 ). The total UES score was significantly higher for device-orientation-based panning compared to drag-based panning. All subscales except Perceived Usability were also significantly higher for device-orientation compared to drag.

However, Perceived Usability showed a tendency to go in the same direction as the other results, and there could be a roof effect. An alpha level of .05 was used for all tests. The effects, measured using Cohen's d, were moderate to large for all significant results. The internal consistency measured using Cronbach's a was excellent or very good on all dimensions. The results of the one-tailed T-tests, Cohen's $d$ and Cronbach's a are shown in the sidebar on the next page. The results provides support for the hypothesis that devicedirection based panning facilitate user engagement.

\begin{tabular}{|l|c|c|c|c|}
\hline & \multicolumn{2}{|c|}{$\begin{array}{c}\text { Drag-based } \\
\text { panning }\end{array}$} & \multicolumn{2}{c|}{$\begin{array}{c}\text { Device- } \\
\text { orientation } \\
\text { based }\end{array}$} \\
\hline \multicolumn{1}{|c|}{ Category } & Mean & \multicolumn{1}{c|}{ SD } & Mean & SD \\
\hline Total UES & 4.45 & 0.71 & 5.11 & 0.78 \\
\hline $\begin{array}{l}\text { Perceived } \\
\text { Usability }\end{array}$ & 5.42 & 1.08 & 5.90 & 1.01 \\
\hline Novelty & 4.58 & 1.46 & 5.18 & 1.11 \\
\hline Felt Involvement & 4.26 & 1.21 & 4.90 & 1.27 \\
\hline $\begin{array}{l}\text { Focused } \\
\text { Attention }\end{array}$ & 2.92 & 0.97 & 4.01 & 1.50 \\
\hline Endurability & 4.29 & 1.26 & 4.95 & 1.24 \\
\hline Aesthetics & 5.42 & 0.96 & 5.78 & 0.65 \\
\hline
\end{tabular}

Table 1. Table 1. Means and standard deviations of the UES questionnaire $(\mathrm{N}=19)$. 


\section{Results of Statistic Tests}

Total UES, $t(18)=3.45$,

$p<.001 ; d=.79 ; a=.92$

Perceived Usability,

$t(18)=1.47, \mathrm{p}>.05$;

$. a=.84$

Novelty, $t(18)=2.27$

$p<.05 ; d=.52 ; a=.89$

Felt Involvement,

$t(18)=2.50, p<.05$;

$d=.58 ; a=.90$

Focused Attention,

$t(18)=4.15, p<.001$;

$d=.95 ; a=.91$

Endurability, $t(18)=2.90$,

$p<.01 ; d=.66 ; \mathrm{a}=.91$

Aesthetics, $t(18)=2.62$,

$p<.01 ; d=.60 ; a=.85$

\section{Acknowledgments}

We wish to thank test participants, Erik Hansson and Phanikumar Kottamasu for their contributions. The Marcus and Amalia Wallenberg Foundation has supported the research through the project The Virtual World Meets the Authentic World in Sensuous and Integrated Learning.
In total, a small majority ( 11 of $19,58 \%$ ) of the participants preferred the device-orientation-based panning and while the rest ( 8 of $19,42 \%$ ) preferred the drag-based panning. In the open-ended questions, users noted that device-orientation was more fun and exciting because of novelty, but also made it easier to shut other things out. It was thought of as more real with a better flow and involvement. The edges of the cubical panorama were experienced as awkward in the device-orientation-based panning. Other issues were that it was difficult to show others the panorama, it looked silly, and it was also experienced as tiresome and cumbersome since large movements were required. Drag-based panning was experienced as more intuitive and familiar, and the participants felt more in control. There was in the application an issue with a slow automatic movement back to the original position of the panorama; some users experienced this as annoying in the drag-based condition.

\section{Conclusions}

The results of this study suggest that device-orientation based panning on handheld devices is useful when engagement (as defined in the UES) is considered important. It should, however, only be used if movement-based interaction is not awkward in the particular context of use [2]. Given the different preferences of our participants, a recommendation would be to support both device-orientation-based panning and drag-based panning. The results of this study also strengthen the idea that more intensive bodily interaction can be more engaging [1]. On-site panoramas with semantic and visual connection to the environment are also likely to be even more engaging, but that is an issue for future research.

\section{References}

[1] Bianchi-Berthouze, N., Kim, W.W., and Darshak, P. Does body movement engage you more in digital game play? and why? In Paiva, A.C.R., Prada, R., and Picard, R.W. (Eds.), ACII 2007, LNCS, vol. 4738. Springer, Heidelberg, 2007, 102-113.

[2] Hürst, W., and Bilyalov, T. (2010). Dynamic versus Static Peephole Navigation of VR Panoramas on Handheld Devices. Proc. In MUM 2010, ACM Press (2010), Article No. 25.

[3] Lehtinen, V., Nurminen, A., \& Oulasvirta, A. Integrating spatial sensing to an interactive mobile 3D map. In Proc. 3DUI 2012, IEEE (2012), 11-14.

[4] Liestol, G., \& Morrison, A. Views, alignment and incongruity in indirect augmented reality. In Proc. ISMAR-AMH, IEEE (2013), 23-28.

[5] Mehra, S., Werkhoven, P., \& Worring, M.

Navigating on handheld displays: Dynamic versus static peephole navigation. ACM Transactions on ComputerHuman Interaction 13, 4 (2006), 448-457.

[6] Mulloni, A., Seichter, H., Dünser, A., Baudisch, P. \& Schmalstieg, D. $360^{\circ}$ panoramic overviews for location-based services. In Proc. CHI 2012, ACM Press (2012), 2565-2568.

[7] Nilsson, S., Arvola, M., Szczepanski, A, and Bång, M. Exploring Place and Direction: Mobile Augmented Reality in the Astrid Lindgren Landscape. In Proc. OzCHI 2012, ACM Press, (2012), 411-419.

[8] O'Brien, H. L., and Toms, E. G. Is there a universal instrument for measuring interactive information retrieval?: the case of the user engagement scale. In Proc. IIiX 2010, ACM Press (2010), 335-340.

[9] Wither, J., Tsai, Y. T., \& Azuma, R. Indirect augmented reality. Computers \& Graphics 35, 5 (2011), 810-822. 